\title{
Flavor Mixing, CP-Violation and the Masses of the Light Quarks
}

\author{
H. Fritzsch ${ }^{\mathrm{a}} \mathrm{b}$

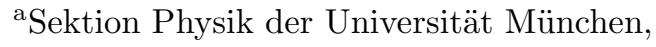 \\ Theresienstrasse 37, D-80333 München \\ ${ }^{\mathrm{b}}$ CERN, Route de Meyrin 385, 1211 Geneva 23, Switzerland
}

The observed hierarchy of the quark masses is interpreted as a signal for an underlying "subnuclear democracy" as the relevant symmetry of the quark mass terms. A simple breaking of the symmetry leads to a mixing between the second and the third family, in agreement with observation. Introducing the mixing between the first and the second family, one finds an interesting pattern of maximal $C P$-violation as well as a complete determination of the elements of the CKM matrix and of the unitarity triangle.

My talk at this conference on QCD is dealing with the important topic of flavor mixing, which is a topic outside QCD. However, it is strongly linked to QCD by the quark masses. In QCD the quark masses are merely free parameters, like the electron mass in QED. However, a detailed knowledge of the dynamics of QCD is required to deduce the mass eigenvalues from the experimental data. It is likely that the quark mass eigenvalues, actually rather ratios of those, determine the weak mixing angles. Thus the quark masses serve as a bridge between chromodynamics and flavor dynamics.

In the standard electroweak model both the masses of the quarks as well as the weak mixing angles enter as free parameters, given by the corresponding Yukawa coupling constants. Any further insight into the yet unknown dynamics of mass generation would imply a step beyond the physics of the electroweak standard model. At present it seems far too early to attempt an actual solution of the dynamics of mass generation, and one is invited to follow a strategy similar to the one which led eventually to the solution of the strong interaction dynamics by QCD, by looking for specific patterns and symmetries as well as specific symmetry violations in the internal flavor space of the quarks and leptons.

The mass spectra of the quarks are dominated largely by the masses of the members of the third family, i. e. by $t$ and $b$. Thus a clear hierarchical pattern exists. Furthermore the masses of the first family are small compared to those of the second one. Moreover, the CKM-mixing matrix also exhibits a hierarchical pattern - the transitions between the second and third family as well as between the first and the third family are small compared to those between the first and the second family.

About 15 years ago, it was emphasized ${ }^{1)}$ that the observed hierarchies signify that nature seems to be close to the so-called "rank-one" limit, in which all mixing angles vanish and both the $\mathrm{u}^{-}$ and d-type mass matrices are proportional to the rank-one matrix

$M_{0}=$ const. $\cdot\left(\begin{array}{lll}0 & 0 & 0 \\ 0 & 0 & 0 \\ 0 & 0 & 1\end{array}\right)$.

Whether the dynamics of the mass generation allows that this limit can be achieved in a consistent way remains an unsolved issue, depending on the dynamical details of mass generation. Here we take the point of view that the quark mass eigenvalues are dynamical entities, and one could change their values in order to study certain symmetry limits, as it is done in QCD. In the standard electroweak model, in which the quark mass matrices are given by the coupling of a scalar field to the various quark field, this can certainly be done by changing the related-coupling constants. 
Whether it is possible at all in reality remains to be seen.

It is well-known that the quark mass matrices can always be made hermitean by a suitable transformation of the righthanded fields. We shall suppose in this paper that the quark mass matrices are hermitean. The limit described by eq. (1) is a non-trivial constraint; it can be derived from imposing a chiral symmetry, as emphasized in ref. (2). This symmetry ensures that an electroweak doublet which is massless remains unmixed and is coupled to the $W$-boson with full strength.

As soon as the mass is introduced, at least for one member of the doublet, the symmetry is violated and mixing phenomena are expected to show up. That way a chiral evolution of the CKM matrix can be considered. ${ }^{2}$ ) At the first stage only the $t$ and $b$ quark masses are introduced, due to their non-vanishing coupling to the scalar "Higgs" field. The CKM-matrix is unity in this limit. At the next stage the second generation acquires a mass. Since the $(u, d)$-doublet is still massless, only the second and the third generations mix, and the CKM-matrix is given by a real $2 \times 2$ rotation matrix in the $(c, s)-(t, b)$ subsystem, describing e. g. the mixing between $s$ and $b$.

In the limit where the masses of the $u$ and $d$ quarks are set to zero, the quark mass matrices $M_{i j}$ both for the charge $2 / 3$ and charge $-1 / 3$ quarks can be arranged such that all elements $M_{i j}$ and $M_{i j}(i=1,2,3)$ are zero.

Thus the quark mass matrices have the form:

$M_{i j}=\left(\begin{array}{ccc}0 & 0 & 0 \\ 0 & a & b \\ 0 & b^{*} & C\end{array}\right)$

The observed mass hierarchy is incorporated into this structure by denoting the entry which is of the order of the $t-$ or $b$-mass by $C$, which $a,|b|<<C$. It can easily be seen (see, e. g. ref. (3)) that the complex phases in the matrices given in e. g. (1) can be rotated away by subjecting both $M_{i j}^{u}$ and $M_{i j}^{d}$ to the same unitary transformation. Thus we shall take $b$ to be real both for $U$-quarks and for $D$-quarks. As expected,
$C P$-violation cannot arise at this stage.

Only at the next step, at which the $u$ and $d$ masses are introduced, does the full CKM-matrix appear, described in general by three angles and one phase, and only at this step $C P$-violation can appear. Thus it is the generation of mass for the first family which is responsible for the violation of $C P$-symmetry.

It has been emphasized some time ago ${ }^{4,5)}$ that the rank-one mass matrix (see eq. (1)) can be expressed in terms of a "democratic mass matrix":

$M_{0}=c\left(\begin{array}{lll}1 & 1 & 1 \\ 1 & 1 & 1 \\ 1 & 1 & 1\end{array}\right)$,

which exhibits an $S(3)_{L} \times S(3)_{R}$ symmetry. Writing down the mass eigenstates in terms of the eigenstates of the "democratic" symmetry, one finds e.g. for the $u$-quark channel:

$$
\begin{aligned}
u^{0} & =\frac{1}{\sqrt{2}}\left(u_{1}-u_{2}\right) \\
c^{0} & =\frac{1}{\sqrt{6}}\left(u_{1}+u_{2}-2 u_{3}\right) \\
t^{0} & =\frac{1}{\sqrt{3}}\left(u_{1}+u_{2}+u_{3}\right) .
\end{aligned}
$$

Here $u_{1}, \ldots$ are the symmetry eigenstates. Note that $u^{0}$ and $c^{0}$ are massless in the limit considered here, and any linear combination of the first two state vectors given in eq. (3) would fulfill the same purpose, i. e. the decomposition is not unique, only the wave function of the coherent state $t^{0}$ is uniquely defined. This ambiguity will disappear as soon as the symmetry is violated.

The wave functions given in eq. (3) are reminiscent of the wave functions of the neutral pseudoscalar mesons in QCD in the $S U(3)_{L} \times$ $S U(3)_{R}$ limit:

$$
\begin{aligned}
\pi_{0}^{0} & =\frac{1}{\sqrt{2}}(\bar{u} u-\bar{d} d) \\
\eta_{0} & =\frac{1}{\sqrt{6}}(\bar{u} u+\bar{d} d-2 \bar{s} s) \\
\eta_{0}^{\prime} & =\frac{1}{\sqrt{3}}(\bar{u} u+\bar{d} d+\bar{s} s) .
\end{aligned}
$$

(Here the lower index denotes that we are considering the chiral limit). Also the mass spectrum 
of these mesons is identical to the mass spectrum of the quarks in the "democratic" limit: two mesons $\left(\pi_{0}^{0}, \eta_{0}\right)$ are massless and act as NambuGoldstone bosons, while the third coherent state $\eta_{0}^{\prime}$ is not massless due to the QCD anomaly.

In the chiral limit the (mass) ${ }^{2}$-matrix of the neutral pseudoscalar mesons is also a "democratic" mass matrix when written in terms of the $(\bar{q} q)$ - eigenstates $(\bar{u} u),(\bar{d} d)$ and $(\bar{s} s)^{6)}$ :

$M^{2}(p s)=\lambda\left(\begin{array}{lll}1 & 1 & 1 \\ 1 & 1 & 1 \\ 1 & 1 & 1\end{array}\right)$

where the strength parameter $\lambda$ is given by $\lambda=$ $M^{2}\left(\eta_{0}^{\prime}\right) / 3$. The mass matrix (6) is the dynamical consequence of the QCD-anomaly which causes strong transitions between the quark eigenstates (due to gluonic annihilation effects enhanced by topological effects). Likewise one may argue that analogous transitions are the reason for the lepton-quark mass hierarchy. Here we shall not speculate about a detailed mechanism of this type, but merely study the effect of symmetry breaking.

In the case of the pseudoscalar mesons the breaking of the symmetry down to $S U(2)_{L} \times$ $S U(2)_{R}$ is provided by a direct mass term $m_{s} \bar{s} s$ for the s-quark. This implies a modification of the $(3,3)$ matrix element in eq. (5), where $\lambda$ is replaced by $\lambda+M^{2}(\bar{s} s)$ where $M^{2}(\bar{s} s)$ is given by $2 M_{K}^{2}$, which is proportional to $<\bar{s} s>_{0}$, the expectation value of $\bar{s} s$ in the QCD vacuum. This direct mass term causes the violation of the symmetry and generates at the same time a mixing between $\eta_{0}$ and $\eta_{0}^{\prime}$, a mass for the $\eta_{0}$, and a mass shift for the $\eta_{0}^{\prime}$.

It would be interesting to see whether an analogue of the simplest violation of this kind of symmetry violation of the "democratic" symmetry which describes successfully the mass and mixing pattern of the $\eta-\eta^{\prime}$-system is also able to describe the observed mixing and mass pattern of the second and third family of leptons and quarks. This was discussed recently ${ }^{7)}$. Let us replace the $(3,3)$ matrix element in eq. (2) by $1+\varepsilon_{i} ;(\mathrm{i}=\mathrm{u}$ ( $\mathrm{u}$-quarks), d (d-quarks) respectively. The small real parameters $\varepsilon_{i}$ describe the departure from democratic symmetry and lead a) to a generation of mass for the second family and

b) to a flavour mixing between the third and the second family. Since $\varepsilon$ is directly related (see below) to a fermion mass and the latter is not restricted to be positive, $\varepsilon$ can be positive or negative. (Note that a negative Fermi-Dirac mass can always be turned into a positive one by a suitable $\gamma_{5}$ transformation of the spin $\frac{1}{2}$ field). Since the original mass term is represented by a symmetric matrix, we take $\varepsilon$ to be real.

In ref. [5] a general breaking of the flavor democracy was discussed in term of two parameters $\alpha$ and $\beta$. The ansatz discussed here, in analogy to the case of the pseudoscalar mesons which represents the simplest breaking of the flavor democracy, corresponds to the special case $\alpha=0$. Note that the case $\beta=\alpha+\alpha^{*}$ discussed in ref. [4] leads to the mass matrix given in ref. [1].

It is instructive to rewrite the mass matrix in the hierarchical basis, where one obtains in the case of the down-type quarks:

$M=c_{l}\left(\begin{array}{ccc}0 & 0 & 0 \\ 0 & +\frac{2}{3} \varepsilon_{u} & -\frac{\sqrt{2}}{3} \varepsilon_{u} \\ 0 & -\frac{\sqrt{2}}{3} \varepsilon_{u} & 3+\frac{1}{3} \varepsilon_{u}\end{array}\right)$.

In lowest order of $\varepsilon$ one finds the mass eigenvalues $m_{s}=\frac{2}{9} \varepsilon_{d} \cdot m_{b}, m_{b}=m_{b^{0}}, \Theta_{s, b}=\left|\sqrt{2} \cdot \varepsilon_{d} / 9\right|$.

The exact mass eigenvalues and the mixing angle are given by:

$$
\begin{aligned}
m_{1} / c_{d} & =\frac{3+\varepsilon_{d}}{2}-\frac{3}{2} \sqrt{1-\frac{2}{9} \varepsilon_{d}+\frac{1}{9} \varepsilon_{d}^{2}} \\
m_{2} / c_{d} & =\frac{3+\varepsilon_{d}}{2}+\frac{3}{2} \sqrt{1-\frac{2}{9} \varepsilon_{d}+\frac{1}{9} \varepsilon_{d}^{2}} \\
\sin \Theta_{(s, b)} & =\frac{1}{\sqrt{2}}\left(1-\frac{1-\frac{1}{9} \varepsilon_{d}}{\left(1-\frac{2}{9} \varepsilon_{d}+\frac{1}{9} \varepsilon_{d}^{2}\right)^{1 / 2}}\right)^{1 / 2} .
\end{aligned}
$$

The ratio $m_{s} / m_{b}$ is allowed to vary in the range $0.022 \ldots 0.044$ (see ref. (8)). According to eq. (7) one finds $\varepsilon_{d}$ to vary from $\varepsilon_{d}=0.11$ to 0.21 . The associated $s-b$ mixing angle varies from $\Theta(s, b)=1.0^{\circ} \quad(\sin \Theta=0.018)$ and $\Theta(s, b)=$ 
$1.95^{\circ} \quad(\sin \Theta=0.034)$. As an illustrative example we use the values $m_{b}(1 \mathrm{GeV})=5200 \mathrm{MeV}$, $m_{s}(1 \mathrm{GeV})=220 \mathrm{MeV}$. One obtains $\varepsilon_{d}=0.20$ and $\sin \Theta(s, b)=0.032$.

To determine the amount of mixing in the $(c, t)$-channel, a knowledge of the ratio $m_{c} / m_{t}$ is required. As an illustrative example we take $m_{c}(1 \mathrm{GeV})=1.35 \mathrm{GeV}, m_{c}\left(m_{t}\right)=$ $0.880 \mathrm{GeV}, m_{c} / m_{t}=0.005$. In this case one finds $\varepsilon_{u}=0.023$ and $\Theta(c, t)=0.21^{\circ} \quad(\sin \Theta(c, t)=$ $0.004)$.

The actual weak mixing between the third and the second quark family is combined effect of the two family mixings described above. The symmetry breaking given by the $\varepsilon$-parameter can be interpreted, as done in eq. (7), as a direct mass term for the $u_{3}, d_{3}$ fermion. However, a direct fermion mass term need not be positive, since its sign can always be changed by a suitable $\gamma_{5^{-}}$ transformation. What counts for our analysis is the relative sign of the $m_{s}$-mass term in comparison to the $m_{c}$-term, discussed previously. Thus two possibilities must be considered:

a) Both the $m_{s}-$ and the $m_{c}$-term have the same relative sign with respect to each other, i. e. both $\varepsilon_{d}$ and $\varepsilon_{u}$ are positive, and the mixing angle between the second and third family is given by the difference $\Theta(s b)-\Theta(c t)$. This possibility seems to be ruled out by experiment, since it would lead to $V_{c b}<0.03$.

b) The relative signs of the breaking terms $\varepsilon_{d}$ and $\varepsilon_{u}$ are different, and the mixing angle between the $(s, b)$ and $(c, t)$ systems is given by the sum $\Theta(s b)+\Theta(c t)$. Thus we obtain $V_{c b} \cong \sin (\Theta(s b)+\Theta(c t))$.

According to the range of values for $m_{s}$ discussed above, one finds $V_{c b} \cong 0.022 \ldots 0.038$. For example, for $m_{s}(1 \mathrm{GeV})=220 \mathrm{MeV}$, $m_{c}(1 \mathrm{GeV})=1.35 \mathrm{GeV}$, one obtains $V_{c b} \cong 0.036$.

The experiments give $V_{c b}=0.032 \ldots 0.048^{9}$ ). We conclude from the analysis given above that our ansatz for the symmetry breaking reproduces the lower part of the experimental range. Nevertheless we obtain consistency with experiment only if the ratio $m_{s} / m_{b}$ is relatively large implying $m_{s}(1 \mathrm{GeV}) \geq 180 \mathrm{MeV}$. Note that recent estimates of $m_{s}(1 \mathrm{GeV})$ give values in the range 180 ... $200 \mathrm{MeV}^{10)}$.

It is remarkable that the simplest ansatz for the breaking of the "democratic symmetry", one which nature follows in the case of the pseudoscalar mesons, is able to reproduce the experimental data on the mixing between the second and third family. We interpret this as a hint that the eigenstates of the symmetry, not the mass eigenstates, play a special rôle in the physics of flavour, a rôle which needs to be investigated further.

The next step is to introduce the mass of the $d$ quark, but keeping $m_{u}$ massless. We regard this sequence of steps as useful due to the fact that the mass ratios $m_{u} / m_{c}$ and $m_{u} / m_{t}$ are about one order of magnitude smaller than the ratios $m_{d} / m_{s}$ and $m_{d} / m_{b}$ respectively. It is well-known that the observed magnitude of the mixing between the first and the second family can be reproduced well by a specific texture of the mass matrix $[11$, 12]. We shall incorporate this here and take the following ansatz for the mass matrix of the downtype quarks:

$M_{\mathrm{d}}=\left(\begin{array}{ccc}0 & D_{\mathrm{d}} & 0 \\ D_{\mathrm{d}}^{*} & C_{\mathrm{d}} & B_{\mathrm{d}} \\ 0 & B_{\mathrm{d}} & A_{\mathrm{d}}\end{array}\right)$.

The consideration above with respect to the breaking of the democratic symmetry suggest that $C_{d} / B_{d}=-\sqrt{2}$. However, for our subsequent consideration this specific ratio is not essential. At this stage the mass matrix of the uptype quarks remains in the form (6). The CKM matrix elements $V_{u s}, V_{c d}$ and the ratios $V_{u b} / V_{c b}$, $V_{t d} / V_{t s}$ can be calculated in this limit. One finds in lowest order:

$$
\begin{aligned}
& V_{u s} \approx \sqrt{\frac{m_{d}}{m_{s}}}, \quad V_{c d} \approx \sqrt{\frac{m_{d}}{m_{s}}}, \\
& \frac{V_{u b}}{V_{c b}} \approx 0, \quad \frac{V_{t d}}{V_{t s}} \approx \sqrt{\frac{m_{d}}{m_{s}}} .
\end{aligned}
$$

An interesting implication of the ansatz (8) is the vanishing of $C P$ violation. Although the mass 
matrix (5) contains a complex parameter $D_{d}$, its phase can be rotated away due to the fact that $m_{u}$ is still massless, and a phase rotation of the $u$-field does not lead to any observable consequences. The vanishing of $C P$ violation can be seen as follows. Considering two hermitian mass matrices $M_{\mathrm{u}}$ and $M_{\mathrm{d}}$ in general, one may define a commutator like

$\left[M_{\mathrm{u}}, M_{\mathrm{d}}\right]=i \mathcal{C}$

and prove that its determinant Det $\mathcal{C}$ is a rephasing invariant measure of $C P$ violation [13]. It can easily be checked that Det $\mathrm{C}$ vanishes. The vanishing of $C P$ violation in our approach in the limit $m_{u} \rightarrow 0$ is an interesting phenomenon, since it is the same limit in which the "strong" $C P$ violation induced by instanton effects of QCD is absent [14]. Whether this link between "strong" and "weak" $C P$ violation could offer a solution of the "strong" $C P$ problem remains an open issue at the moment. Nevertheless it is an interesting feature of our approach that $C P$ violation and the mass of the $u$ quark are intrinsically linked to each other. Since the phase of $D$ can be rotated away, it will be disregarded, and $D$ is taken to be real.

The final step is to introduce the mass of the $u$ quark. The mass matrix $M_{\mathrm{u}}$ takes the form:

$M_{\mathrm{u}}=\left(\begin{array}{ccc}0 & D_{\mathrm{u}} & 0 \\ D_{\mathrm{u}}^{*} & C_{\mathrm{u}} & B_{\mathrm{u}} \\ 0 & B_{\mathrm{u}} & A_{\mathrm{u}}\end{array}\right)$

(Here $A_{u}$ etc. are defined analogously as in e.g. (8)). Once the mixing term $D_{\mathrm{u}}=\left|D_{\mathrm{u}}\right| e^{i \sigma}$ for the $u$-quark is introduced, $C P$ violation appears. For the determinant of the commutator $(6)$ we find ${ }^{15)}$ :

Det $\mathcal{C} \cong T \sin \sigma$,

$$
\begin{aligned}
T= & 2\left|D_{\mathrm{u}} D_{\mathrm{d}}\right|\left[\left(A_{\mathrm{u}} B_{\mathrm{d}}-B_{\mathrm{u}} A_{\mathrm{d}}\right)^{2}\right. \\
& -\left|D_{\mathrm{u}}\right|^{2} B_{\mathrm{d}}^{2}-B_{\mathrm{u}}^{2}\left|D_{\mathrm{d}}\right|^{2} \\
& \left.-\left(A_{\mathrm{u}} B_{\mathrm{d}}-B_{\mathrm{u}} A_{\mathrm{d}}\right)\left(C_{\mathrm{u}} B_{\mathrm{d}}-B_{\mathrm{u}} C_{\mathrm{d}}\right)\right] .
\end{aligned}
$$

The phase $\sigma$ determines the strength of $C P$ violation. The diagonalization of the mass matrices $M_{\mathrm{d}}$ and $M_{\mathrm{u}}$ leads to theigenvalues $m_{i}(i=$ $u, d, \ldots)$. Note that $m_{u}$ and $m_{d}$ appear to be negative. By a suitable $\gamma_{5}$-transformation of the quark fields one can arrange them to be positive. Collecting the lowest order terms in the CKM matrix, one obtains:

$$
\begin{aligned}
& V_{u s} \approx \sqrt{\frac{m_{d}}{m_{s}}}-\sqrt{\frac{m_{u}}{m_{c}}} e^{i \sigma}, \\
& V_{c d} \approx \sqrt{\frac{m_{u}}{m_{c}}}-\sqrt{\frac{m_{d}}{m_{s}}} e^{i \sigma}
\end{aligned}
$$

and

$\frac{V_{u b}}{V_{c b}} \approx-\sqrt{\frac{m_{u}}{m_{c}}}, \quad \frac{V_{t d}}{V_{t s}} \approx-\sqrt{\frac{m_{d}}{m_{s}}}$.

The relations for $V_{u s}$ and $V_{c d}$ were obtained previously [12]. However then it was not noted that the relative phase between the two ratios might be relevant for $C P$ violation. A related discussion can be found in ref. [16].

According to eq. (12) the strength of $C P$ violation depends on the phase $\sigma$. If we keep the modulus of the parameter $D_{\mathrm{u}}$ constant, but vary the phase from zero to $90^{\circ}$, the strength of $C P$ violation varies from zero to a maximal value given by eq. (12), which is obtained for $\sigma=90^{\circ}$. We conclude that $C P$ violation is maximal for $\sigma=90^{\circ}$. In this case the element $D_{\mathrm{u}}$ would be purely imaginary, if we set the phase of the matrix element $D_{\mathrm{d}}$ to be zero. As discussed above, this can always be arranged.

In our approach the $C P$-violating phase also enters in the expressions for $V_{u s}$ and $V_{c d}$ (Cabibbo angle). As discussed already in ref. [12], the Cabibbo angle is fixed by the difference of $\sqrt{m_{d} / m_{s}}$ and $\sqrt{m_{u} / m_{c}} \times$ phase factor. The second term contributes a small correction (of order 0.06 ) to the leading term, which according to the mass ratios given in ref. [8] is allowed to vary between 0.20 and 0.24 . For our subsequent discussion we shall use $0.218 \leq\left|V_{u s}\right| \leq 0.224$ [8]. If the phase parameter multiplying $\sqrt{m_{u} / m_{c}}$ were zero or $\pm 180^{\circ}$ (i.e. either the difference or sum of the two real terms would enter), the observed magnitude of the Cabibbo angle could not be reproduced. Thus a phase is needed, and we find within our approach purely on phenomenological grounds that $C P$ violation must be present if we request consistency between observation and our result (14). 
An excellent description of the magnitude of $V_{u s}$ is obtained for a phase angle of $90^{\circ}$. In this case one finds:

$$
\left|V_{u s}\right|^{2} \approx\left(1-\frac{m_{d}}{m_{s}}\right)\left(\frac{m_{d}}{m_{s}}+\frac{m_{u}}{m_{c}}\right),
$$

where approximations are made for $V_{u s}$ to a better degree of accuracy than that in eq. (14). Using $\left|V_{u s}\right|=0.218 \ldots 0.224$ and $m_{u} / m_{c}=$ $0.0028 \ldots 0.0048$ we obtain $m_{d} / m_{s} \approx 0.045 \ldots 0.05$. This corresponds to $m_{s} / m_{d} \approx 20 \ldots 22$, which is entirely consistent with the determination of $m_{s} / m_{d}$, based on chiral perturbation theory [8]: $m_{s} / m_{d}=17 \ldots 25$. This example shows that the phase angle must be in the vicinity of $90^{\circ}$. Fixing $m_{u} / m_{c}$ to its central value and varying $m_{d} / m_{s}$ throughout the allowed range, we find $\sigma \approx 66^{0} \ldots 110^{0}$.

The case $\sigma=90^{\circ}$, favoured by our analysis, deserves a special attention. It implies that in the sequence of steps discussed above the term $D_{\mathrm{u}}$ generating the mass of the $u$-quark is purely imaginary, and hence $C P$ violation is maximal. It is of high interest to observe that nature seems to prefer this case. A purely imaginary term $D_{\mathrm{u}}$ implies that the algebraic structure of the quark mass matrix is particularly simple. Its consequences need to be investigated further and might lead the way to an underlying internal symmetry responsible for the pattern of masses.

Finally we explore the consequences of our approach to the unitarity triangle, i.e., the triangle formed by the CKM matrix elements $V_{u b}^{*}, V_{t d}$ and $s_{12} V_{c b}\left(s_{12}=\sin \theta_{12}, \theta_{12}\right.$ : Cabibbo angle $)$ in the complex plane (we shall use the definitions of the angles $\alpha, \beta$ and $\gamma$ as given in ref. [9). For $\sigma=90^{0}$ we obtain:

$$
\begin{aligned}
& \alpha \approx 90^{\circ}, \quad \beta \approx \arctan \sqrt{\frac{m_{u}}{m_{c}} \cdot \frac{m_{s}}{m_{d}}} \\
& \gamma \approx 90^{\circ}-\beta .
\end{aligned}
$$

Thus the unitarity triangle is a rectangular triangle. We note that the unitarity triangle and the triangle formed in the complex phase by $V_{u s}, \sqrt{m_{d} / m_{s}}$ and $\sqrt{m_{u} / m_{c}}$ are similar rectangular triangles, related by a scale transformation. Using as input $m_{u} / m_{c}=0.0028 \ldots 0.0048$ and $m_{s} / m_{d}=20 \ldots 22$ as discussed above, we find $\beta \approx 13^{0} \ldots 18^{0}, \gamma \approx 72^{0} \ldots 76^{0}$, and $\sin 2 \beta \approx \sin 2 \gamma$ $\approx 0.45 \ldots 0.59$. These values are consistent with the experimental constraints.

We have shown that a simple pattern for the generation of masses for the first family of leptons and quarks leads to an interesting and predictive pattern for the violation of $C P$ symmetry. The observed magnitude of the Cabibbo angle requires $C P$ violation to be maximal or at least near to its maximal strength. The ratio $V_{u b} / V_{c b}$ as well as $V_{t d} / V_{t s}$ are given by $\sqrt{m_{u} / m_{c}}$ and $\sqrt{m_{d} / m_{s}}$ respectively. In the case of maximal $C P$ violation the unitarity triangle is rectangular $\left(\alpha=90^{\circ}\right)$, the angle $\beta$ can vary in the range $13^{0} \ldots 18^{0}(\sin 2 \beta=\sin 2 \gamma \approx 0.45 \ldots 0.59)$. It remains to be seen whether the future experiments, e.g. the measurements of the $C P$ asymmetry in $B$-decays, $B_{d}^{0}$, confirm these values. 


\section{Acknowledgements:}

I should like to thank Prof. S Narison for his efforts in arranging this meeting in this wonderful town in Languedoc-Roussilon.

\section{REFERENCES}

1. H. Fritzsch, Nucl. Phys. B155 (1988) 189; See also: Fritzsch H 1984 in: Proc. Europhysics Conf. on Flavor Mixing,

Erice, Italy, Ling-Li Chau ed.

P. L. Anthony et. al. (E142), Phys. Rev. Lett. 71 (1993)

2. H. Fritzsch, Phys. Lett. B184 (1992) 391.

3. H. Lehmann and T. T. Wu; Phys. Lett. B384 (1996) 249

4. H. Harari, Haut and J. Weyers (1978);

Phys. Lett. B78 459;

A. Chikashige, G. Gelmini, R. P. Peccei and M. Roncadelli (1980) Phys. Lett. 94B 499;

C. Jarlskog (1986) in: Proc. of the Int. Symp. on Production and Decay of

Heavy Flavors, Heidelberg, Germany, P. Kaus and S. Meshkov Phys. Lett. A3 (1988) 1251, A4 (1989) 603

G. C. Branco, J. I. Silva-Marcos and M. N. Rebelo Phys. Lett. B237 (1990) 446.

Y. Koide, Phys. Rev., D28 (1983) 252

K. Kang and X. K. Kang, Phys. Rev. D56 (1997) 1511

5. H. Fritzsch and J. Plankl, Phys. Lett. B237 (1990) 451

6. H. Fritzsch and P. Minkowski, Nuovo Cimento 30A (1975) 393

H. Fritzsch and D. Jackson, Phys. Lett. 66B (1977) 365

7. H. Fritzsch and D. Holtmannspötter, Phys. Lett. B338 (1994) 290

8. J. Gasser and H. Leutwyler, Phys. Rev. 87 (1982) 77

See also: S. Narison, Phys. Lett. B358 (1995) 113

9. Particle Data Group, M. Aguilar-Benitez et al. Phys. Rev. D50 (1994) 1173

10. M. Jamin and M. Münz, Z. Phys. C66 (1995) 633

11. S. Weinberg, Transactions of the New York
Academy of Sciences, Series II, Vol. 38 (1977) 185

12. H. Fritzsch, Phys. Lett. 70B (1977) 436

13. C. Jarlskog, Phys. Ref. Lett. 55 (1984) 1039

14. R. D. Peccei and H. R. Quinn, Phys. Rev. D16 (1977) 1791

S. Weinberg, Phys. Rev. Lett. 40 (1978) 223

KF. Wilczek, Phys. Rev. Lett. 40 (1978) 279

15. H. Fritzsch and Z. Xing Phys. Lett. B353 (1995) 95

16. M. Shin, Phys. Lett. B145 (1984) 285

M. Gronau, R. Johnson and J. Schechter, Phys. Rev. Lett. 54 (1985) 2176 